\title{
Electron Phase Separations Involving Superconductivity in the Extended Hubbard Models with Pair Hopping Interaction
}

\author{
K.J. KAPCIA*
}

Electron States of Solids Division, Faculty of Physics, Adam Mickiewicz University in Poznan, Umultowska 85, 61-614 Poznań, Poland

In this work the extended Hubbard models with pair hopping interaction (at the atomic limit) are investigated within the variational approach, which treats the on-site interaction term exactly and the intersite interactions within the mean-field approximation (exact in $d \rightarrow+\infty$ ). We analyze mutual stability of the superconducting phase and charge or (ferro/antiferro-)magnetic orderings as well as homogeneous mixed phases. Our preliminary results for $U=0$ show that the superconducting phase can coexist with the charge or (ferro/antiferro-)magnetic phases only in states with electron phase separation.

DOI: $10.12693 /$ APhysPolA.127.204

PACS: 71.10.Fd, 74.20.-z, 74.25.Dw, 64.75.Gh, 71.10.Hf

\section{Introduction}

The phase separation (PS) phenomenon involving superconductivity ( $\mathrm{SC}$ ) is under intense investigation and has been evidenced in broad range of currently investigated materials including iron pnictides, cuprates, bismutates, organic conductors and heavy-fermion systems (for review see e.g. Refs. [1-20] and references therein).

The effective model considered has a form of extended Hubbard model at the atomic limit $(t=0)$ :

$$
\begin{array}{r}
\hat{H}=U \sum_{i} \hat{n}_{i \uparrow} \hat{n}_{i \downarrow}-I \sum_{\langle i, j\rangle}\left(\hat{\rho}_{i}^{+} \hat{\rho}_{j}^{-}+\hat{\rho}_{j}^{+} \hat{\rho}_{i}^{-}\right)+\frac{W}{2} \sum_{\langle i, j\rangle} \hat{n}_{i} \hat{n}_{j} \\
-2 J^{z} \sum_{\langle i, j\rangle} \hat{s}_{i}^{z} \hat{s}_{j}^{z}-J^{x y} \sum_{\langle i, j\rangle}\left(\hat{s}_{i}^{+} \hat{s}_{j}^{-}+\hat{s}_{j}^{+} \hat{s}_{i}^{-}\right)-\mu \sum_{i} \hat{n}_{i},
\end{array}
$$

where $\hat{n}_{i \sigma}=\hat{c}_{i \sigma}^{+} \hat{c}_{i \sigma}, \hat{n}_{i}=\sum_{\sigma} \hat{n}_{i \sigma}, \hat{\rho}_{i}^{+}=\left(\hat{\rho}_{i}^{-}\right)^{\dagger}=\hat{c}_{i \uparrow}^{+} \hat{c}_{i \downarrow}^{+}$, $\hat{s}_{i}^{z}=(1 / 2)\left(\hat{n}_{i \uparrow}-\hat{n}_{i \downarrow}\right)$ and $\hat{s}_{i}^{+}=\left(\hat{s}_{i}^{-}\right)^{\dagger}=\hat{c}_{i \uparrow}^{+} \hat{c}_{i \downarrow} \cdot \hat{c}_{i \sigma}^{+}$and $\hat{c}_{i \sigma}$ denote the creation and annihilation operators, respectively, of an electron with $\operatorname{spin} \sigma(\sigma=\uparrow, \downarrow)$ at site $i$. Interactions $U, I, W, J^{z}, J^{x y}$ between the nearest neighbors (NN) are effective model parameters (assumed to include all the possible contributions and renormalizations) and $\mu$ is the chemical potential.

The analysis has been performed within a variational approach (VA) [2-7], which treats the $U$ term exactly and the intersite interactions within the mean-field approximation (MFA), which is a rigorous treatment of the intersite terms in the limit of infinite dimensions $d \rightarrow+\infty$ or large coordination number (number of NN) $z$.

We introduce order parameters defined as: $\Delta_{q}=$ $\frac{1}{N} \sum_{i} \exp \left(\mathrm{i} \boldsymbol{q} \cdot \boldsymbol{r}_{i}\right)\left\langle\hat{\rho}_{i}^{-}\right\rangle, \quad n_{\boldsymbol{q}}=\frac{1}{N} \sum_{i} \exp \left(\mathrm{i} \boldsymbol{q} \cdot \boldsymbol{r}_{i}\right)\left\langle\hat{n}_{i}\right\rangle$, $m_{\boldsymbol{q}}^{\alpha}=\frac{1}{N} \sum_{i} \exp \left(\mathrm{i} \boldsymbol{q} \cdot \boldsymbol{r}_{i}\right)\left\langle\hat{s}_{i}^{\alpha}\right\rangle$ for two sublattice orderings on the alternate lattices, where $N$ is the total number of lattice sites. The model exhibits a symmetry between $I>0$ (SC with $s$-pairing, $\Delta_{\mathbf{0}} \neq 0$ ) and $I<0$ (SC with $\eta$-pairing, $\Delta_{Q} \neq 0, Q$ is a half of the smallest reciprocal lattice vector) cases [3, 4]. Analogical

\footnotetext{
* corresponding author; e-mail: konrad.kapcia@amu.edu.pl
}

symmetry occurs for spin orderings in both direction $(\alpha=z, \pm)$ between ferro- (with $m_{\mathbf{0}}^{\alpha} \neq 0$ ) and antiferro(with $m_{\boldsymbol{Q}}^{\alpha} \neq 0$ ) magnetic (M) phases (for general case of both $J^{x y}$ and $J^{z}$ nonzero that symmetry is only for VA results). The boundary between $\mathrm{M}$ phases with ordering in $z$-direction and $x y$-direction is for $\left|J^{x y} / J^{z}\right|=1$ (within VA). Thus we define $|J|=\operatorname{Max}\left\{\left|J^{z}\right|,\left|J^{x y}\right|\right\}$ and $\left|J_{0}\right|=z|J|$. In the presence of finite single electron hopping $t \neq 0$ the symmetries are broken in the general case and the effects of $t \neq 0$ have been also discussed in Refs. [11-20]. In the charge-ordered (CO) phase $n_{\boldsymbol{Q}} \neq 0$. $n \equiv n_{0}$ is electron concentration in the system. Notice that phase diagrams obtained are symmetric with respect to $n=1\left(\bar{\mu}=0, \bar{\mu}=\mu-U / 2-W_{0}, W_{0}=z W\right)$ because of the particle-hole symmetry of the model.

In this paper we particularly focus on the interplay and competition between pair hopping $(I)$ interaction and intersite magnetic $(J)$ or density-density $(W)$ interactions. The impact of on-site term $U$ will not be analyzed, thus we restrict ourselves to $U=0$ case. Names of transition orders used are consistent with our earlier works [3-8].

\section{Results and discussion for $U=0$}

Let us consider the $U=0$ case of the model analyzed. In such a case, for $|W|,|J|<|I|$ only the second-order transition SC-NO occurs with increasing temperature $T$ and only the $\mathrm{SC}$ phase can be stable in low $T$. The $k_{\mathrm{B}} T /\left|I_{0}\right|$ vs. $n$ phase diagram of the model is shown in Fig. $1\left(I_{0}=z I\right)$. The analytical result for the SC-NO boundary as a function of $n$ can be obtained as $k_{\mathrm{B}} T_{S C} /\left|I_{0}\right|=(n-1) \ln ^{-1}(n /(2-n))[2,3](n<1)$. The structure of the diagram as a function of $\mu$ is the same as of the diagram obtained for $W=0$ and $J=0$ [3]. For $W=0$ and any $J$, on the SC-NO transition line the relation $\bar{\mu} / I_{0}=n-1$ occurs (generally always in the $\mathrm{SC}$ phase), but for $W \neq 0$ the relation $\mu(n)$ is not so simple (cf. Refs. [3, 8, 9, 21-24]).

\subsection{Superconductivity and magnetism}

First, let us discuss the competition between $I$ and $J$ interactions. Following discussion of phase diagrams is 


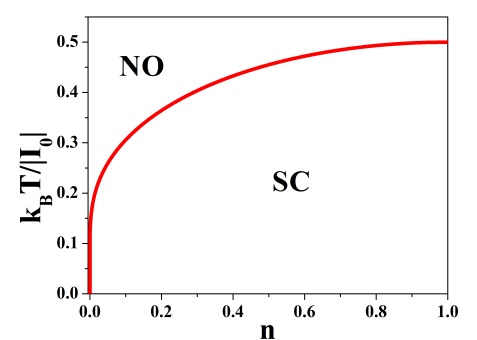

Fig. 1. Phase diagram for $|W|,|J|<|I|, \quad U=0$, $I_{0}=z I$.

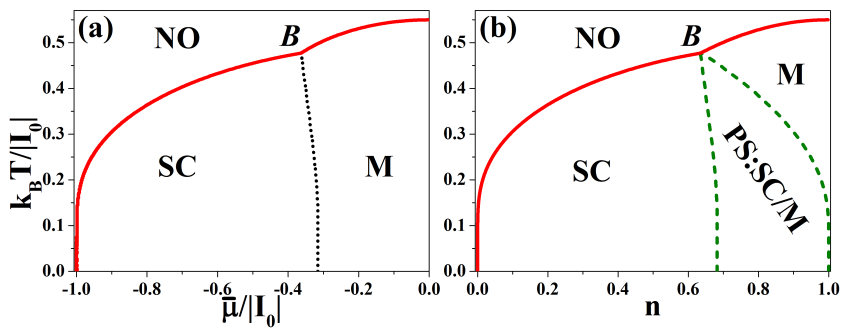

Fig. 2. Phase diagrams for $|J| /|I|=1.1(U=0$, $W=0, \bar{\mu}=\mu-U / 2)$. Details in text.

valid for $|W| /|I|<1$, but $W \neq 0$ changes the thermodynamical characteristics and the locations of phase boundaries only on diagrams as a function of $\mu$ (not as a function of $n$ ).

In particular, the phase diagrams for $|J| /|I|=1.1$ $(W=0)$ are shown in Fig. 2. For that case there are three homogeneous phases ( $\mathrm{SC}, \mathrm{M}, \mathrm{NO}$ ) on the phase diagrams. The transitions $\mathrm{SC}-\mathrm{NO}$ and $\mathrm{M}-\mathrm{NO}$ with increasing temperature are second-order ones and their temperatures decrease with increasing $|\bar{\mu}|$ and $|n-1|$. The analytical equations for temperatures $T_{\mathrm{SC}}$ and $T_{\mathrm{M}}$ of these transition, respectively, as a function of $n$ can be derived (in their ranges of occurrence): for $T_{\mathrm{SC}}$ it was given above in the beginning of Sect. 2, whereas for $T_{\mathrm{M}}$ it is obtained as $k_{\mathrm{B}} T_{\mathrm{M}} /\left|J_{0}\right|=n(2-n) / 2$. The $\mathrm{SC}-\mathrm{M}$ transition is firstorder (for fixed $\mu$ ) and thus the PS:SC/M state is stable in the defined range of $n$. The first-order SC-M as well as the "third-order" SC-PS and PS-M transition temperatures increase with $|\bar{\mu}|$ and $|1-n|$, respectively. All transition lines merge at a bicritical point, denoted as $B$ on the diagrams. Notice that the MIX1:SC/M phase (a coexistence of SC and $\mathrm{M}$ in homogeneous phase, $\Delta_{\boldsymbol{q}}, m_{\boldsymbol{q}}^{\alpha} \neq 0$ ) does not appear on the diagrams at any $T \geq 0$.

For $|J| /|I|=1$ the SC and M phases are degenerated for $n=1(\bar{\mu}=0)$, whereas for $n \neq 1$ the SC phase is stable. The case of $n=1$ for arbitrary $U /\left|I_{0}\right|$ has been analyzed in Ref. [25], whereas the behaviour of the system at $T=0$ have been investigated in Ref. [9]. With increasing $|J|$, the $B$-point moves along the SC-NO boundary toward larger $|\bar{\mu}|(|1-n|)$. The region of the $\mathrm{M}$ phase stability is extended, whereas the region of the $\mathrm{SC}$ phase occurrence is reduced by increasing the ratio $|J| /|I|$. The location of the $B$-point can be determined from the equation $T_{\mathrm{SC}}=T_{\mathrm{M}}$. Moreover, for higher $|J| /|I|$ the structure of the diagram can also change. Exemplarily, for $|J| /|I| \gtrsim 1.34$ the M-NO transition can also be first-order (if it occurs at $k_{\mathrm{B}} T /\left|J_{0}\right|<1 / 3$, for fixed $\mu$ ) [25], the multi- critical point changes its type (there can be more than one multicritical point on the diagram), and the PS:M/NO state can also occur (cf. also [26-28]). For $|J| /|I|>2$ the region of the SC phase occurrence vanishes totally. The detailed analyses of this problem will be explored further in a subsequent publication.

\subsection{Superconductivity and charge orderings}

Next, we will discuss the diagrams of the model for $|W| /|I|>1$ and $|J|<|I|$. The examples of the phase diagrams for $W /|I|=1.1$ and $|J|<|I|)$ are presented in Fig. 3 . For the case $W /|I|>1$ there are three homogeneous phases $(\mathrm{SC}, \mathrm{CO}, \mathrm{NO})$ on the phase diagrams and the structures of the diagrams are similar to those discussed in previous section. The transitions to the NO phase with increasing temperature (i.e. SC-NO, CO-NO) are second-order ones and their temperatures decrease with increasing $|\bar{\mu}|$ and $|n-1|$ [8]. The analytical equations for temperatures $T_{\mathrm{SC}}$ and $T_{\mathrm{CO}}$ of these transition, respectively, as a function of $n$ can be derived (in their ranges of occurrence): for $T_{\mathrm{SC}}$ it was given above in the beginning of Sect. 2, whereas for $T_{\mathrm{CO}}$ it is obtained as $k_{\mathrm{B}} T_{\mathrm{CO}} / W_{0}=n(2-n) / 2$. The transition between two ordered phases is first-order (for fixed $\mu$ ) and the PS:SC/CO state is stable in the define range of $n$. The first-order $\mathrm{SC}-\mathrm{CO}$ as well as the "third-order" SC-PS and PS-CO transition temperatures increase with $|\bar{\mu}|$ and $|1-n|$, respectively. Similar as in previous case all transitions lines merge at a bicritical point $\boldsymbol{B}$. Moreover, at $T=0$ the PS:SC/CO state is degenerated with the MIX2:SC/CO phase (a coexistence of SC and CO in homogeneous phase, $\left.\Delta_{\boldsymbol{q}}, n_{\boldsymbol{Q}} \neq 0\right)$, but at any $T>0$ that degeneration is removed $[8,9,29]$.

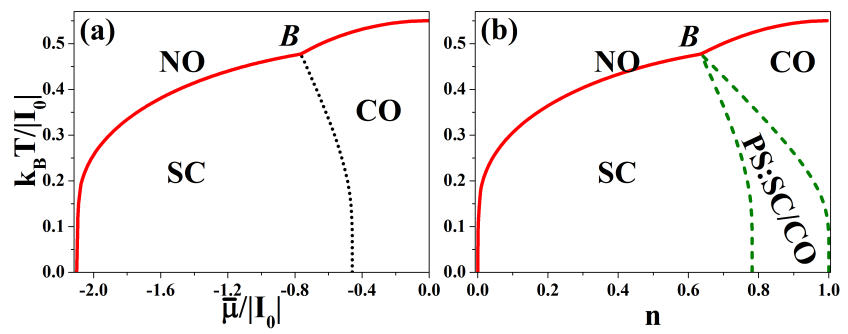

Fig. 3. Phase diagrams for $W /|I|=1.1$ and $|J| /|I|<1$ $\left(U=0, \bar{\mu}=\mu-U / 2-W_{0}, W_{0}=z W\right)$. Details in text.

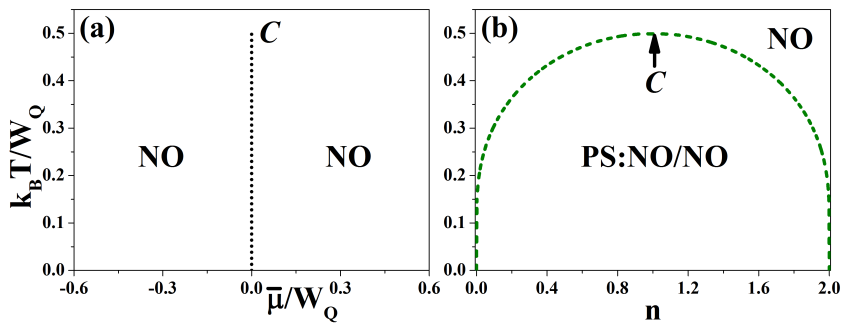

Fig. 4. As Fig. 3 for $W /|I|<-1,|J| /|I|<1$ and $W_{Q}=-z W$.

With increasing $W>0$, the $\boldsymbol{B}$-point moves along the SC-NO boundary toward larger $|\bar{\mu}|(|1-n|)$. Its location can be determined from the equation $T_{\mathrm{SC}}=T_{\mathrm{CO}}$. The region of the CO phase stability is extended, whereas the region of the $\mathrm{SC}$ phase occurrence is reduced, by increasing the ratio $W /|I|$. For $W=|I|$ there homogeneous phases: SC, CO and MIX2 are degenerated at $n=1[1,8,9]$, but the SC phase has a lowest energy for $n \neq 1$. 
For $W /|I|<-1$ only the NO phase and the PS:NO/NO state (in define range of $n$ ) are present on phase diagrams. The diagrams for $W /|I|<-1$ are shown in Fig. 4 (cf. also Refs. $[8,9,24])$ and they do not change with decreasing $W<0$. The first-order NO-NO transition line (vertical one for fixed $\bar{\mu}=0$ ) ends at critical point $\boldsymbol{C}$ located at $k_{\mathrm{B}} T / W_{Q}=$ 0.5. The analytical result for the PS:NO/NO-NO boundary as a function of $n$ can be obtained as $k_{\mathrm{B}} T_{P S} / W_{Q}=(n-$ 1) $\ln ^{-1}(n /(2-n))\left(W_{Q}=-z W, n<1\right)$. For $W /|I|=-1$ the SC phase and the NO phases (the PS:NO/NO state) are degenerated for any $\bar{\mu}$ (for any $n$, respectively).

\section{Conclusions and final remarks}

In conclusions, we note that the analysis of the model considered $(U=0)$ shows that the superconductivity can coexist with magnetism (for $|J| /|I|>1$ ) and charge orderings (for $W /|I|>1$ ) in the states with phase separation (PS:SC/CO and PS:SC/M, respectively) and the mixed homogeneous phases (MIX1, MIX2) do not appear on the phase diagrams at $T>0$. In particular, we have derived that transitions between the superconducting phase and other ordered phases (the $\mathrm{SC}-\mathrm{CO}$ and $\mathrm{SC}-\mathrm{M}$ transitions) are of the first order for fixed chemical potential $\mu$ and it leads to the occurrence of the phase separation (PS:SC/CO and PS:SC/M, respectively) in the definite ranges of electron concentration $n$.

Notice that repulsive $U>0[3,5]$ or external magnetic field $[4,6]$ can change the $\mathrm{SC}-\mathrm{NO}$ transition into first-order one (for fixed $\mu$ ) and the phase separation state PS:SC/NO can occur in the define ranges of $n$. Moreover, $U<0$ favors the SC phase, whereas $U>0$ favors the M phase. The CO phase for $W>0$ as well as the PS:NO/NO state for $W<0$ can exist for both signs of $U$ and sufficiently large $|W| /|I|$ (if $U \gg 0$ these states can be stable only for $n \neq 1$ ), cf. e.g. Refs. [21-24].

In this paper we have not considered the case of $|J|>|I|$ and $|W|>|I|$. The analysis of the interplay between $J$ and $W$ interactions [30-32] is beyond the goal of this work and will be presented elsewhere.

\section{Acknowledgments}

The author thanks S. Robaszkiewicz and R. Micnas for fruitful discussions and a careful reading of the manuscript. The work has been financed in part by National Science Center (Poland) as grant No. DEC-2011/01/N/ST3/00413 and doctoral scholarship No. DEC-2013/08/T/ST3/00012 as well as from ESF - OP "Human Capital" — POKL.04.01.01-00$133 / 09-00$

\section{References}

[1] R. Micnas, J. Ranninger, S. Robaszkiewicz, Rev. Mod. Phys. 62, 113 (1990).

[2] S. Robaszkiewicz, G. Pawłowski, Physica C 210, 61 (1993).

[3] K. Kapcia, S. Robaszkiewicz, R. Micnas, J. Phys. Condens. Matter 24, 215601 (2012).

[4] K. Kapcia, S. Robaszkiewicz, J. Phys. Condens. Matter 25, 065603 (2013).

[5] K.J. Kapcia, J. Supercond. Nov. Magn. 27, 913 (2014).

[6] K.J. Kapcia, Acta Phys. Pol. A 126, A-53 (2014).

[7] K.J. Kapcia, Ph.D. thesis, Adam Mickiewicz University in Poznań, Poznań 2014.

[8] K. Kapcia, J. Supercond. Nov. Magn. 26, 2647 (2013); A. Ptok, K.J. Kapcia, arXiv:1502.00579, Supercond. Sci. Technol. in press (2015).

[9] K.J. Kapcia, J. Supercond. Novel Magn. in press (2015); DOI:10.1007/s10948-014-2906-4.
[10] R. Micnas, J. Ranninger, S. Robaszkiewicz, J. Phys. C Solid State Phys. 21, L145 (1988).

[11] S. Robaszkiewicz, B. Bułka, Phys. Rev. B 59, 6430 (1999); W.R. Czart, S. Robaszkiewicz, Phys. Rev. B 64, 104511 (2001).

[12] G.I. Japaridze, A.P. Kampf, M. Sekania, P. Kakashvili, Ph. Brune, Phys. Rev. B 65 014518 (2001).

[13] S. Robaszkiewicz, W.R. Czart, Acta Phys. Pol. B 32, 3267 (2001); W.R. Czart, S. Robaszkiewicz, Acta Phys. Pol. A 106, 709 (2004); W.R. Czart, S. Robaszkiewicz, Acta Phys. Pol. A 109, 577 (2006).

[14] W.R. Czart, S. Robaszkiewicz, Phys. Status Solidi B 243, 151 (2006); W.R. Czart, S. Robaszkiewicz, B. Tobijaszewska, Acta Phys. Pol. A 114, 129 (2008); W.R. Czart, P.R. Grzybowski, M. Nogala and S. Robaszkiewicz, Acta. Phys. Pol. A 121, 1042 (2012).

[15] C. Dziurzik, G.I. Japaridze, A. Schadschneider, J. Zittartz, Eur. Phys. J. B 37, 453 (2004).

[16] A. Ptok, M.M. Maśka, M. Mierzejewski, J. Phys. Condens. Matter 21, 295601 (2009).

[17] A. Ptok, M.M. Maśka, M. Mierzejewski, Phys Rev. B 84, 094526 (2011).

[18] A. Ptok, D. Crivelli, K.J. Kapcia, Supercond. Sci. Technol. 28, 045010 (2015).

[19] A. Ptok, K.J. Kapcia, arXiv:1502.00579, Supercond Sci. Technol. 28, 045022 (2015).

[20] S. Robaszkiewicz, Phys. Status Solidi B 59, K63 (1973); W.R. Czart, S. Robaszkiewicz, Material Science - Poland 25, 485 (2007).

[21] K. Kapcia, W. Kłobus, S. Robaszkiewicz, Acta Phys. Pol. A 118, 350 (2010); K. Kapcia, S. Robaszkiewicz,Acta Phys. Pol. A 121, 1029 (2012).

[22] K. Kapcia, S. Robaszkiewicz, J. Phys. Condens. Matter 23, 105601 (2011).

[23] K. Kapcia, S. Robaszkiewicz, J. Phys. Condens. Matter 23, 249802 (2011).

[24] R.J. Bursill, C.J. Thompson, J. Phys. A Math. Gen 26, 4497 (1993).

[25] K. Kapcia, Acta Phys. Pol. A 121, 733 (2012).

[26] W. Kłobus, K. Kapcia, S. Robaszkiewicz, Acta Phys. Pol. A 118, 353 (2010).

[27] S. Murawski, K. Kapcia, G. Pawłowski, S. Robaszkiewicz, Acta Phys. Pol. A $\mathbf{1 2 1}$ 1035 (2012)

[28] S. Murawski, K.J. Kapcia, G. Pawłowski, S. Robaszkiewicz, Acta Phys. Pol. A 126, A-110 (2014); S. Murawski, K.J. Kapcia, G. Pawłowski, S. Robaszkiewicz, Acta Phys. Pol. A 127, 281 (2015).

[29] S. Robaszkiewicz, G. Pawłowski, Acta Phys. Pol. A 90, 569 (1996).

[30] S. Robaszkiewicz, Phys. Status Solidi B 70, K51 (1975).

[31] K. Kapcia, W. Kłobus, S. Robaszkiewicz, Acta Phys. Pol. A 121, 1032 (2012); K.J. Kapcia, W. Kłobus, S. Robaszkiewicz, Acta Phys. Pol. A 127, 284 (2015).

[32] F. Mancini, E. Plekhanov, G. Sica, J. Phys. Conf. Series 391, 012148 (2012). 